\title{
Perfis das produções científicas elaboradas pela enfermagem durante o primeiro ano
} da pandemia da COVID-19

\author{
Profiles of scientific productions prepared by nursing during the first year of the COVID-19 \\ pandemic
}

Perfiles de producciones científicas elaboradas por enfermería durante el primer año de la pandemia COVID-19

\author{
Sabrina Iracema da Silva Couto \\ ORCID: https://orcid.org/0000-0002-3864-4843 \\ Universidade Maurício de Nassau, Brasil \\ E-mail: sabrinacouto89@gmail.com \\ Maria Aparecida Guimarães da Silva \\ ORCID: https://orcid.org/0000-0001-7364-1173 \\ Universidade Maurício de Nassau, Brasil \\ E-mail: enfa.aguimaraes@gmail.com \\ Maria Júlia Souza Marques \\ ORCID: https://orcid.org/0000-0002-1136-2926 \\ Universidade Maurício de Nassau, Brasil \\ E-mail: juliascc@hotmail.com \\ Laryssa Grazielle Feitosa Lopes \\ ORCID: https://orcid.org/0000-0002-0709-5378 \\ Universidade Maurício de Nassau, Brasil \\ Faculdade de Medicina do Sertão, Brasil \\ E-mail: lara_grazi@hotmail.com \\ Laís de Macêdo Ferreira Santos \\ ORCID: https://orcid.org/0000-0003-1834-7961 \\ Universidade Maurício de Nassau, Brasil \\ E-mail: santos.laismf@gmail.com
}

\begin{abstract}
Resumo
Objetivo: Traçar os perfis das produções científicas elaboradas por profissionais ou acadêmicos brasileiros de enfermagem durante o primeiro ano da pandemia da COVID-19. Metodologia: Trata-se de uma revisão integrativa da literatura, realizada no mês de agosto de 2020, nas bases de dados Biblioteca Virtual em Saúde (BVS), Literatura Latino-Americana e do Caribe em Ciências da Saúde (LILACS), Google Scholar e Scientific Eletronic Library OnLine (SCIELO). Foram utilizados artigos escritos na língua portuguesa, elaborados por profissionais ou acadêmicos de enfermagem, brasileiros, publicados no período entre janeiro de 2020 a dia 23 de agosto de 2020 , sendo em revistas nacionais ou internacionais. Resultados: Foram encontrados 480 artigos no total, após rigor metodológico por meio dos critérios de inclusão e exclusão, foram selecionados 25 artigos, sendo 7 artigos na LILACS, 1 na BVS, 3 no SCIELO e 14 no Google Acadêmico. A análise dos artigos foi realizada de forma descritiva e predispôs a etapa de extração dos dados: base de dados, autores/mês de publicação, título/revista, tipo de artigo e categoria de tema. Conclusão: Diante dos resultados encontrados na literatura pode-se observar que a enfermagem é uma profissão que está ligada diretamente com o cuidado assistencial integral ao paciente, sendo uma das categorias de profissionais da saúde que mais se destacou dentro do cenário da pandemia, por ser os trabalhadores da saúde que mais estavam em contato com pacientes infectados pelo coronavírus, e no meio desse cenário constitui-se o desafio da construção de conhecimento científico sobre essa doença.
\end{abstract}

Palavras-chave: Enfermagem; Coronavírus; COVID-19; Pandemia.

\begin{abstract}
Objective: Tracing the profiles of scientific productions prepared by Brazilian nursing professionals or academics during the first year of the COVID-19 pandemic. Methodology: This is an integrative literature review, carried out in August 2020, in the Virtual Health Library (VHL), Latin American and Caribbean Literature in Health Sciences (LILACS), Google Scholar and Scientific Electronic Library OnLine (SCIELO). Articles written in Portuguese, written by Brazilian nursing professionals or academics, published in the period between January 2020 and August 23, 2020, in national or international journals, were used. Results: A total of 480 articles were found, after methodological rigor through the inclusion and exclusion criteria, 25 articles were selected, being 7 articles in
\end{abstract}


LILACS, 1 in the VHL, 3 in SCIELO and 14 in Google Scholar. The analysis of the articles was performed descriptively and predisposed the data extraction stage: database, authors/month of publication, title/magazine, type of article and topic category. Conclusion: Based on the results found in the literature, it can be observed that nursing is a profession that is directly linked to comprehensive patient care, being one of the categories of health professionals that most stood out within the pandemic scenario, as it is the health workers who were most in contact with patients infected by the coronavirus, and in the midst of this scenario, the challenge of building scientific knowledge about this disease is constituted.

Keywords: Nursing; Coronaviruses; COVID-19; Pandemic.

\section{Resumen}

Objetivo: Seguimiento de los perfiles de producciones científicas elaboradas por profesionales o académicos de enfermería brasileños durante el primer año de la pandemia COVID-19. Metodología: Se trata de una revisión integradora de la literatura, realizada en agosto de 2020, en la Biblioteca Virtual en Salud (BVS), Literatura Latinoamericana y del Caribe en Ciencias de la Salud (LILACS), Google Scholar y Biblioteca Electrónica Científica en Línea (SCIELO). Se utilizaron artículos escritos en portugués, escritos por profesionales o académicos de enfermería brasileños, publicados en el período comprendido entre enero de 2020 y el 23 de agosto de 2020, en revistas nacionales o internacionales. Resultados: Se encontraron un total de 480 artículos, luego de rigor metodológico mediante los criterios de inclusión y exclusión, se seleccionaron 25 artículos, siendo 7 artículos en LILACS, 1 en la BVS, 3 en SCIELO y 14 en Google Scholar. El análisis de los artículos se realizó de forma descriptiva y predispuso la etapa de extracción de datos: base de datos, autores / mes de publicación, título / revista, tipo de artículo y categoría temática. Conclusión: con base en los resultados encontrados en la literatura, se puede observar que la enfermería es una profesión que está directamente vinculada a la atención integral al paciente, siendo una de las categorías de profesionales de la salud que más se destacó dentro del escenario pandémico, como es el trabajadores de la salud que estuvieron más en contacto con los pacientes infectados por el coronavirus, y en medio de este escenario, se constituye el desafío de construir conocimiento científico sobre esta enfermedad.

Palabras clave: Enfermería; Coronavirus; COVID-19; Pandemia.

\section{Introdução}

Em dezembro de 2019 na cidade de Wuhan na China, foi encontrado o vírus Sars-Cov-2, até então desconhecido, sendo ele apenas um em meio a sete subtipos capazes de desenvolver doenças na espécie humana (Barbosa, Gomes \& Gomes, 2020). A semelhança com outros vírus respiratórios, como o causador da Síndrome Respiratória Aguda Grave (SARS) e Síndrome Respiratória no Oriente Médio (MERS) contribuiu para sua descoberta (Pedrosa \& Albuquerque, 2020; Silva, dos Santos \& Melo, 2020).

Inicialmente seu descobrimento se deu ao aumento de pacientes internados com quadro de pneumonia e evolução para insuficiência respiratória aguda grave de contaminação rápida e elevados números de morte, se fazendo necessário que o governo da China comunicasse a Organização Mundial de Saúde (OMS) desse surto. Com isso, através de estudos foi possível constatar que o causador de tal surto possuía um agente etiológico de origem zoonótica, o nomeando assim de Sars-Cov-2, responsável pela pandemia da Coronavírus Disease 2019 (COVID-19) anunciada em março de 2020 (Barbosa, Gomes \& Gomes, 2020; Pedrosa \& Albuquerque, 2020).

A disseminação da COVID-19 se dá através de gotículas do trato respiratório. Com o intuito de diminuir sua propagação, a OMS recomendou que todos os países afetados pela doença aderissem ao isolamento social e uso de equipamentos de proteção individual (EPI) (Cheng et al., 2020; Dantas et al., 2020).

Embora ainda não se saiba sobre todas as complicações futuras da doença, o paciente acometido inicialmente irá apresentar sinais e sintomas semelhantes ao de uma síndrome gripal, como febre, tosse seca e dores de garganta, como também pode apresentar falta de ar, um sintoma característico da COVID-19 em pacientes mais críticos, podendo haver complicações e evoluir para síndromes respiratórias graves, principalmente em pacientes dos grupos de risco, onde estão inclusos diabéticos, hipertensos, idosos, obesos e portadores de outras doenças que o torne imunodeprimido (Marins et al., 2020; Machado et al., 2020). 
Diante desse cenário de desafios e incertezas, especialistas estão a todo momento analisando informações e investindo na produção científica, visando desenvolver vacinas e medidas de controle para o novo coronavírus. Para uma informação confiável e de relevância, os pesquisadores de áreas como: microbiologia, infectologia, biologia, psicologia, virologia, enfermagem e entre outros, se dedicam incansavelmente aos estudos e pesquisas a fim de contribuir para a ciência acerca da COVID-19, sendo a enfermagem um campo de destaque para tratar desse assunto, uma vez que é uma das áreas da linha de frente, com total assistência ao paciente positivo para a doença (Dantas et al., 2020; Costa et al., 2020; da Silva et al., 2020).

Dentro desse contexto da pandemia e seus impactos, o objetivo é compreender e traçar os perfis das produções científicas elaboradas por profissionais ou acadêmicos brasileiros de enfermagem durante o primeiro ano da pandemia da Covid-19, bem como contribuir com a Literatura Brasileira.

\section{Metodologia}

Esta pesquisa trata-se de uma revisão integrativa da literatura, que é um método utilizado para investigar estudos já existentes com intuito de se obter resultados sobre um determinado tema, de forma ordenada e sintetizado. Para a elaboração da revisão, foram utilizados os procedimentos metodológicos recomendados pela literatura vigente, sendo eles: 1) Identificação do tema e da questão norteadora; 2) Estabelecimento de critérios de inclusão e exclusão; 3) Categorização dos artigos; 4) Avaliação dos resultados incluídos; 5) Interpretação dos resultados; 6) Síntese do conhecimento (Botelho et al., 2011; Souza et al., 2010).

A realização desta pesquisa pautou-se na seguinte questão norteadora: quais os perfis e os comportamentos das produções científicas elaboradas pelos profissionais ou acadêmicos de enfermagem brasileiros durante o ano de 2020 da pandemia da COVID-19?

Após rigor metodológico, as bases de dados utilizadas foram a Biblioteca Virtual em Saúde (BVS), Literatura LatinoAmericana e do Caribe em Ciências da Saúde (LILACS), Google Scholar e Scientific Eletronic Library OnLine (SCIELO). Para as bases de dados foram usados os descritores em Ciências da Saúde (DeCS): "Enfermagem", "Coronavírus", "COVID19" e "Pandemia". Visando atingir os objetivos almejados, os descritores foram utilizados de forma isolada e de forma combinada.

O levantamento foi realizado no mês de agosto de 2020, atendendo a alguns critérios de inclusão e exclusão. Foram excluídos trabalhos incompletos, artigos que não cumpriam o período estipulado, ensaios teóricos, relatos de experiência, artigos de opinião, resumos estendidos, artigos elaborados por outros profissionais que não sejam da área de Enfermagem, artigos de reflexão e trabalhos que não compreendiam a temática estudada. Foram incluídos na pesquisa apenas estudos completos, sendo artigos originais ou revisões da literatura, que atendam ao objetivo desta pesquisa, escritos na língua portuguesa, elaborados por profissionais ou acadêmicos de enfermagem, brasileiros, publicados no período entre janeiro de 2020 a dia 23 de agosto de 2020, sendo em revistas nacionais ou internacionais.

\section{Resultados}

Foram encontrados 480 artigos no total, após rigor metodológico por meio dos critérios de inclusão e exclusão, foram selecionados 25 artigos, sendo 7 artigos na LILACS, 1 na BVS, 3 no SCIELO e 14 no Google Acadêmico, a forma de seleção descrita pode ser observada na Figura 1. 
Figura 1: Esquema da seleção dos artigos.
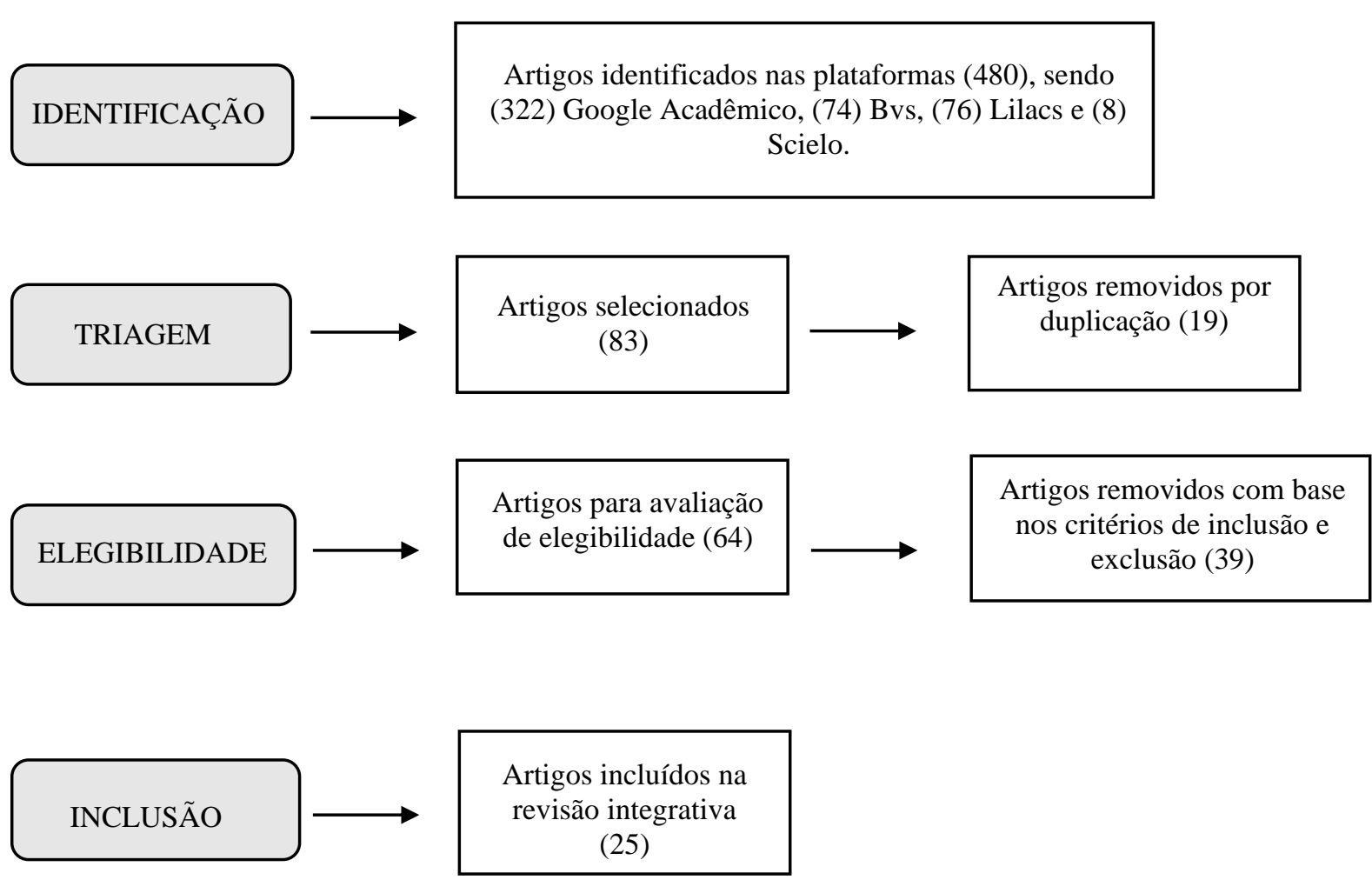

Artigos incluídos na revisão integrativa (25)

Fonte: Autores (2020).

A análise dos artigos foi feita de forma descritiva e predispôs a etapa de extração dos dados: base de dados, autores/mês de publicação, título/revista, tipo de artigo e categoria de tema (Quadro 1).

Quadro 1 - Classificação dos artigos.

\begin{tabular}{|c|c|c|c|c|}
\hline Base de dados & $\begin{array}{c}\text { Autores/mês de } \\
\text { publicação }\end{array}$ & Título/Revista & $\begin{array}{c}\text { Tipo de } \\
\text { artigo } \\
\end{array}$ & Categoria de tema \\
\hline $\begin{array}{l}\text { Google } \\
\text { Acadêmico }\end{array}$ & $\begin{array}{l}\text { da Silva et al. / } \\
\text { Abril }\end{array}$ & $\begin{array}{l}\text { Análise bibliométrica da produção científica sobre Coronavírus e } \\
\text { Covid-19 / Revista Saúde Coletiva (Barueri) }\end{array}$ & $\begin{array}{ll}\text { Revisão da } \\
\text { literatura }\end{array}$ & Produção Científica \\
\hline SCIELO & $\begin{array}{l}\text { Forte \& Pires / } \\
\text { Abril }\end{array}$ & $\begin{array}{l}\text { Os apelos da enfermagem nos meios de comunicação em tempos } \\
\text { de coronavírus / Revista Brasileira de Enfermagem }\end{array}$ & Original & $\begin{array}{l}\text { Atuação da enfermagem } \\
\text { e demais profissionais } \\
\text { de saúde }\end{array}$ \\
\hline $\begin{array}{l}\text { Google } \\
\text { Acadêmico }\end{array}$ & Imoto et al. / Abril & $\begin{array}{l}\text { Cloroquina e Hidroxicloroquina no tratamento da COVID-19: } \\
\text { Sumário de Evidências / Revista Comunicação em Ciências da } \\
\text { Saúde }\end{array}$ & $\begin{array}{ll}\text { Revisão } & \text { da } \\
\text { literatura }\end{array}$ & $\begin{array}{l}\text { Cloroquina } \\
\text { Hidroxicloroquina }\end{array}$ \\
\hline $\begin{array}{l}\text { Google } \\
\text { Acadêmico }\end{array}$ & $\begin{array}{lr}\text { Pedrosa } & \& \\
\text { Albuquerque } & / \\
\text { Abril } & \\
\end{array}$ & $\begin{array}{l}\text { Análise Espacial dos Casos de COVID-19 e leitos de terapia } \\
\text { intensiva no estado do Ceará, Brasil / Revista Ciência \& Saúde } \\
\text { Coletiva }\end{array}$ & Original & $\begin{array}{l}\text { Análise de fatores } \\
\text { relacionados ao SARS- } \\
\text { CoV-2 e pandemia }\end{array}$ \\
\hline $\begin{array}{l}\text { Google } \\
\text { Acadêmico }\end{array}$ & $\begin{array}{l}\text { Rondelli et al. I } \\
\text { Abril }\end{array}$ & $\begin{array}{l}\text { Assistência às gestantes e recém-nascidos no contexto da infecção } \\
\text { covid-19: uma revisão sistemática / Revista Desafios }\end{array}$ & $\begin{array}{ll}\text { Revisão } \\
\text { literatura }\end{array}$ & $\begin{array}{l}\text { Saúde das Gestantes, } \\
\text { Recém-nascidos e } \\
\text { Crianças }\end{array}$ \\
\hline $\begin{array}{l}\text { Google } \\
\text { Acadêmico }\end{array}$ & $\begin{array}{l}\text { Silva, dos Santos } \\
\& \text { Melo / Abril }\end{array}$ & $\begin{array}{l}\text { Aspectos da infecção ocasionada pelo Coronavírus da Síndrome } \\
\text { Respiratória Aguda Grave } 2 \text { (SARS-CoV-2) / Revista Brazilian of } \\
\text { health Review }\end{array}$ & $\begin{array}{ll}\text { Revisão } \\
\text { literatura }\end{array}$ & $\begin{array}{l}\text { Análise de fatores } \\
\text { relacionados ao SARS- } \\
\text { CoV-2 e pandemia }\end{array}$ \\
\hline $\begin{array}{l}\text { Google } \\
\text { Acadêmico }\end{array}$ & $\begin{array}{l}\text { Sousa Júnior et al. } \\
\text { / Abril }\end{array}$ & $\begin{array}{l}\text { "\#Fiqueemcasa e cante comigo": estratégia de entretenimento } \\
\text { musical durante a pandemia de covid-19 no Brasil / Revista UFRR } \\
\text { BOLETIM DE CONJUNTURA (BOCA) }\end{array}$ & Original & Saúde Mental \\
\hline $\begin{array}{l}\text { Google } \\
\text { Acadêmico }\end{array}$ & $\begin{array}{l}\text { Barbosa, Gomes } \\
\text { \& Gomes / Maio }\end{array}$ & $\begin{array}{l}\text { Fatores de estresse nos profissionais de enfermagem no combate à } \\
\text { pandemia da COVID-19: Síntese de Evidências / Revista } \\
\text { Comunicação em Ciências da Saúde }\end{array}$ & $\begin{array}{ll}\text { Revisão } & \text { da } \\
\text { literatura } & \end{array}$ & Saúde Mental \\
\hline SCIELO & $\begin{array}{l}\text { Cheng et al. / } \\
\text { Maio }\end{array}$ & $\begin{array}{l}\text { Comportamento da produção científica sobre síndrome respiratória } \\
\text { aguda grave / Revista Ciências da Saúde }\end{array}$ & $\begin{array}{ll}\text { Revisão da } \\
\text { literatura }\end{array}$ & Produção Científica \\
\hline $\begin{array}{l}\text { Google } \\
\text { Acadêmico }\end{array}$ & $\begin{array}{l}\text { de Souza et al. I } \\
\text { Maio }\end{array}$ & $\begin{array}{l}\text { Hidroxicloroquina como alternativa para o tratamento da infecção } \\
\text { causada pelo SARS-CoV-2: O que se sabe até agora? / Revista } \\
\text { Brazilian of health Review }\end{array}$ & $\begin{array}{l}\text { Revisão } \quad \text { da } \\
\text { literatura }\end{array}$ & $\begin{array}{l}\text { Cloroquina } \\
\text { Hidroxicloroquina }\end{array}$ \\
\hline Google & Schneider et al. / & Utilização de têxteis impregnados com antimicrobianos nos & Revisão & Atuação da enfermagem \\
\hline
\end{tabular}


Research, Society and Development, v. 11, n. 1, e56411125165, 2022

(CC BY 4.0) | ISSN 2525-3409 | DOI: http://dx.doi.org/10.33448/rsd-v11i1.25165

\begin{tabular}{|c|c|c|c|c|}
\hline Acadêmico & Maio & serviços de saúde: revisão integrativa / Revista Ciência \& Saúde & literatura & $\begin{array}{l}\text { e demais profissionais } \\
\text { de saúde }\end{array}$ \\
\hline $\begin{array}{l}\text { Google } \\
\text { Acadêmico }\end{array}$ & Silva et al. / Maio & $\begin{array}{l}\text { Desafios na condução de pesquisas sobre a COVID19 envolvendo } \\
\text { seres humanos no Brasil / Revista Comunicação em Ciências da } \\
\text { Saúde }\end{array}$ & $\begin{array}{lr}\text { Revisão } & \text { da } \\
\text { literatura } & \end{array}$ & Produção Científica \\
\hline $\begin{array}{l}\text { Google } \\
\text { Acadêmico }\end{array}$ & $\begin{array}{l}\text { Soares et al. / } \\
\text { Maio }\end{array}$ & $\begin{array}{l}\text { Pandemia da covid-19: pesquisa documental a partir de } \\
\text { publicações do conselho federal de enfermagem } \\
\text { Revista Enfermagem em Foco }\end{array}$ & Original & Produção Científica \\
\hline SCIELO & $\begin{array}{llll}\text { Costa } & \text { et al. I } \\
\text { Junho } & & \end{array}$ & $\begin{array}{l}\text { Produção científica em periódicos online sobre o novo coronavírus } \\
\text { (COVID-19): pesquisa bibliométrica / Revista Texto \& Contexto } \\
\text { Enfermagem }\end{array}$ & $\begin{array}{l}\text { Revisão } \\
\text { literatura }\end{array}$ & Produção Científica \\
\hline LILACS & $\begin{array}{l}\text { Dantas et al. / } \\
\text { Junho }\end{array}$ & $\begin{array}{l}\text { Diagnósticos de enfermagem para pacientes com COVID-19 / } \\
\text { Revista Journal Health NPEPS }\end{array}$ & $\begin{array}{l}\text { Revisão } \\
\text { literatura }\end{array}$ & $\begin{array}{l}\text { Atuação da enfermagem } \\
\text { e demais profissionais } \\
\text { de saúde }\end{array}$ \\
\hline LILACS & $\begin{array}{l}\text { Machado et al. I } \\
\text { Junho }\end{array}$ & $\begin{array}{l}\text { Parada cardiorrespiratória na pandemia por coronavírus: revisão } \\
\text { compreensiva da literatura / Revista Enfermagem UERJ }\end{array}$ & $\begin{array}{l}\text { Revisão } \\
\text { literatura }\end{array}$ & $\begin{array}{l}\text { Análise de fatores } \\
\text { relacionados ao SARS- } \\
\text { CoV-2 e pandemia }\end{array}$ \\
\hline $\begin{array}{l}\text { Google } \\
\text { Acadêmico }\end{array}$ & $\begin{array}{l}\text { Pacheco et al. I } \\
\text { Junho }\end{array}$ & $\begin{array}{l}\text { Recomendações para o cuidado à criança frente ao novo } \\
\text { coronavírus / Revista Cogitare Enfermagem }\end{array}$ & $\begin{array}{l}\text { Revisão } \\
\text { literatura }\end{array}$ & $\begin{array}{l}\text { Saúde das Gestantes, } \\
\text { Recém-nascidos } \\
\text { Crianças }\end{array}$ \\
\hline BVS & $\begin{array}{l}\text { de Souza Neto \& } \\
\text { Freitas / Julho }\end{array}$ & $\begin{array}{l}\text { Utilização de máscaras: indicações de uso e manejo durante a } \\
\text { pandemia da covid-19/ Revista Cogitare Enfermagem }\end{array}$ & $\begin{array}{l}\text { Revisão } \\
\text { literatura }\end{array}$ & $\begin{array}{l}\text { Análise de fatores } \\
\text { relacionados ao SARS- } \\
\text { CoV-2 e pandemia }\end{array}$ \\
\hline $\begin{array}{l}\text { Google } \\
\text { Acadêmico }\end{array}$ & $\begin{array}{l}\text { Gandra et al. I } \\
\text { Julho }\end{array}$ & $\begin{array}{l}\text { Fatores de riscos assistenciais relacionados a contaminação de } \\
\text { profissionais de enfermagem por Covid-19: Uma revisão da } \\
\text { literatura / Revista Brazilian Journal of Developmen }\end{array}$ & $\begin{array}{l}\text { Revisão } \\
\text { literatura }\end{array}$ & $\begin{array}{l}\text { Atuação da enfermagem } \\
\text { e demais profissionais } \\
\text { de saúde }\end{array}$ \\
\hline $\begin{array}{l}\text { Google } \\
\text { Acadêmico }\end{array}$ & $\begin{array}{l}\text { Marins et al. I } \\
\text { Julho }\end{array}$ & $\begin{array}{l}\text { Enfermeiro na linha de frente ao COVID-19: A experiência da } \\
\text { realidade vivenciada / Revista Research, Society and Development }\end{array}$ & Original & Saúde Mental \\
\hline LILACS & $\begin{array}{l}\text { de Lima et al. / } \\
\text { Agosto }\end{array}$ & $\begin{array}{l}\text { A Imagem do Enfermeiro no Instagram no Contexto da Pandemia } \\
\text { da Covid-19 / Revista Enfermagem em Foco }\end{array}$ & Original & $\begin{array}{l}\text { Análise de fatores } \\
\text { relacionados ao SARS- } \\
\text { CoV-2 e pandemia }\end{array}$ \\
\hline LILACS & $\begin{array}{l}\text { de Sousa Júnior et } \\
\text { al. / Agosto }\end{array}$ & $\begin{array}{l}\text { Pandemia do coronavírus: estratégias amenizadoras do estresse } \\
\text { ocupacional em trabalhadores da saúde / Revista Enfermagem em } \\
\text { Foco }\end{array}$ & $\begin{array}{l}\text { Revisão } \\
\text { literatura }\end{array}$ & Saúde Mental \\
\hline LILACS & $\begin{array}{l}\text { do Nascimento et } \\
\text { al. / Agosto }\end{array}$ & $\begin{array}{l}\text { Impacto da COVID-19 sob o trabalho da enfermagem brasileira: } \\
\text { aspectos epidemiológicos / Revista Enfermagem em Foco }\end{array}$ & Original & $\begin{array}{l}\text { Análise de fatores } \\
\text { relacionados ao SARS- } \\
\text { CoV-2 e pandemia }\end{array}$ \\
\hline LILACS & $\begin{array}{l}\text { Geremia et al. / } \\
\text { Agosto }\end{array}$ & $\begin{array}{l}\text { Pandemia COVID-2019: formação e atuação da enfermagem para } \\
\text { o Sistema Único de Saúde / Revista Enfermagem em Foco }\end{array}$ & Original & $\begin{array}{l}\text { Atuação da enfermagem } \\
\text { e demais profissionais } \\
\text { de saúde }\end{array}$ \\
\hline LILACS & $\begin{array}{l}\text { Moreira \& de } \\
\text { Lucca / Agosto }\end{array}$ & $\begin{array}{l}\text { Apoio psicossocial e saúde mental dos profissionais de } \\
\text { enfermagem no combate ao covid-19 / Revista Enfermagem em } \\
\text { Foco }\end{array}$ & $\begin{array}{l}\text { Revisão } \\
\text { literatura }\end{array}$ & Saúde Mental \\
\hline
\end{tabular}

Fonte: Autores (2020).

Analisando os dados do quadro, observa-se que dos 25 artigos utilizados, 12 foram elaborados por Enfermeiros formados (48,0\%), 5 por acadêmicos de Enfermagem (20,0\%) e 8 por autores misto - formados e acadêmicos (32,0\%). Nota-se que 15 artigos foram elaborados por autores só de Enfermagem (60,0\%) e 10 por autores multidisciplinares (40,0\%). Evidenciou-se a publicação de 24 artigos em revistas nacionais $(96,0 \%)$ e 1 em revistas internacionais $(4,0 \%)$. Bem como observa-se também que, devido um dos critérios de inclusão ter sido as publicações no ano de 2020, constatou-se quanto aos meses que, janeiro, fevereiro e março não tiveram nenhuma (0,0\%), abril teve $7(28,0 \%)$, maio teve $6(24,0 \%)$, junho teve 4 (16,0\%), julho teve $3(12,0 \%)$ e agosto teve $5(20,0 \%)$. E por fim, outro dado observado são os tipos de estudos, onde 17 artigos são revisões da literatura (68\%) e 8 são artigos originais (32\%).

\section{Discussão}

Mediante a análise dos dados foi possível evidenciar e traçar os perfis das publicações e as suas contribuições para a saúde. E levando em consideração que as publicações abordam temas diferentes, optou-se por discuti-las nas seguintes categorias para melhor compreensão desses perfis. 


\subsection{Análise de fatores relacionados ao SARS-CoV-2 e pandemia}

Atualmente denominada COVID-19, esta doença causada pelo novo coronavírus (Sars-Cov-2) originada na província de Wuhan, China, no final de 2019 se expandiu rapidamente pelo mundo (Pedrosa \& Albuquerque, 2020; do Nascimento, Espinosa, da Silva, Freire \& Terças-Trettel, 2020). Embora a OMS só tenha decretado estado de pandemia apenas em 11 de março de 2020, os casos começaram a surgir em meados de dezembro de 2019 e sua disseminação se tornou emergência de saúde pública de importância internacional em 30 de janeiro de 2020 (Machado et al., 2020; de Lima et al., 2020; de Sousa Neto \& Freitas, 2020).

No século XXI, pelo menos três vírus do grupo coronavírus desencadearam sérios problemas na saúde pública, sendo o Sars-Cov-2 um vírus de terceira geração que tem como estrutura molecular um formato esférico ou elíptico e pleomórfico, com diâmetros que pode chegar entre 60 a 140 mm e material genético de RNA de fita simples, ele tem vida média estimada de aproximadamente 5,6 horas em aço inoxidável e 6,8 horas em plástico, podendo permanecer até 72 horas. Esse vírus vem sendo responsável por números alarmantes de doentes e mortos em todo o mundo (Silva et al., 2020; de Sousa Neto \& Freitas, 2020). Essa mortalidade se dá através de sua alta letalidade, evidenciada por desconforto cardiorrespiratório, devido ao dano alveolar que em alguns pacientes pode evoluir para pneumonia, Síndrome do Desconforto Respiratório Agudo (SDRA), lesão miocárdica, arritmias ventriculares, choque e disfunção de múltiplos órgãos (Pedrosa \& Albuquerque, 2020; Silva, dos Santos \& Melo, 2020; Machado et al., 2020).

Em menos de três meses a pandemia apresentou um número de infectados exorbitantes espalhados por países de todos os continentes, onde sua rápida transmissão se dá de humano para humano mesmo na ausência de sintomas, através de gotículas respiratórias infecciosas (Pedrosa \& Albuquerque, 2020; Silva et al., 2020; de Sousa Neto \& Freitas, 2020). Para conter a sua disseminação faz-se importante seguir as três medidas clássicas de saúde pública, já utilizadas há muito tempo no controle de epidemias, são elas: isolamento de contaminados, quarentena e distanciamento social, essas medidas culminaram em consequências devastadoras para a humanidade, apresentando um clima de instabilidade socioeconômica (Pedrosa \& Albuquerque, 2020; de Lima et al., 2020; de Sousa Neto \& Freitas, 2020).

\subsection{Atuação da enfermagem e demais profissionais de saúde}

Dentro do contexto da pandemia, as equipes de saúde, pesquisadores da área e autoridades sanitárias se encontram na linha de frente para o enfrentamento da situação de saúde, sendo a enfermagem a categoria que constitui mais da metade dessa força de trabalho no Brasil, atuando para promover a saúde da população e cuidados aos pacientes com agravos (Geremia et al., 2020; Forte \& Pires, 2020).

Diante do caráter contagioso que o cenário da pandemia apresenta, é indispensável a utilização dos Equipamentos de Proteção Individual (EPI's) pelos profissionais de saúde. Tal condição potencializa a necessidade do enfrentamento dos desafios relacionados às condições de trabalho, a disponibilidade de insumos em quantidades necessárias e qualificação dos profissionais de saúde para o manejo correto desses insumos. E dentro do ambiente hospitalar as práticas de biossegurança são fundamentais para o controle de infecções, principalmente as cruzadas, pois no manejo assistencial os profissionais precisam se atentar que além dos jalecos, os demais têxteis utilizados pelos pacientes nos serviços de saúde representam uma importante fonte de contaminação (Schneider et al., 2020; Gandra et al., 2020).

Os profissionais de enfermagem estão em contato direto com os pacientes e são responsáveis por gerenciar todo o período assistencial, sendo assim, a Sistematização da Assistência de Enfermagem (SAE) se torna uma importante aliada do enfermeiro, a qual é fundamental na prática clínica assistencial e essencial para o processo de trabalho de toda a equipe de enfermagem, com ênfase na atualização do manejo a cada nova avaliação do quadro clínico do paciente (Dantas et al., 2020). E dentro das competências de enfermagem, destaca-se a educação em saúde, que é de suma importância, tendo seu embasamento 
na cultura, no conhecimento e nas experiências anteriores das pessoas, precisando estar alinhada com os aspectos éticos e legais da profissão (Forte \& Pires, 2020).

Evidencia-se que no tangente a mortalidade dos profissionais de enfermagem pela COVID-19, o Brasil apresenta cada vez mais números maiores que os outros países, principalmente devido à natureza do seu trabalho estando expostos a diversos riscos ocupacionais, os quais podem causar-lhes adoecimento e morte (Gandra et al., 2020).

\subsection{Saúde Mental}

A pandemia trouxe histeria e paranoia para o público em geral, em surtos o medo é comum e pode levar a um comportamento que oscila podendo atingir a todos, e para a COVID-19 isso é ainda mais perceptível uma vez que ainda não se sabe tudo e muito se especula. Na China, local que surgiu a disseminação do coronavírus, o Ministério da Saúde já atentava para os efeitos psicológicos da pandemia, não só dos profissionais de saúde, mas da população geral, onde identificaram um nível significativo de angústia, problemas psicológicos, incluindo ansiedade, depressão e estresse, sendo este mais alto para os enfermeiros, uma vez que os mesmos tinham a sensação de perda de controle da situação, receio pela própria saúde e pela propagação do vírus (Barbosa et al., 2020; Marins et al., 2020; Moreira \& Lucca, 2020).

Os hospitais estão sobrecarregados com o atendimento aos pacientes infectados pelo vírus, resultando em maiores demandas dos profissionais atuantes na linha de frente e sendo possível fonte geradora de estresse. Os profissionais de saúde, são descritos como a categoria populacional mais afetada psicologicamente tendo em vista que experimentam fatores estressores adicionais tais como: aumento da carga de trabalho, medo de contaminar os familiares e também de se contaminar, e raiva do governo e dos sistemas de saúde. A falta de apoio, comunicação e de treinamento são fatores de risco que aumentam a possibilidade do desenvolvimento de doenças psicológicas (Barbosa et al., 2020; Marins et al., 2020; de Sousa Júnior et al., 2020).

As características da profissão de enfermagem requerem que estes profissionais permaneçam um maior tempo ao lado dos pacientes, colocando-os como linha de frente no combate a esta doença. Nesse contexto, a enfermagem brasileira está enfrentando grandes desafios, sem precedentes históricos em sua atuação, nos diversos cenários da assistência à saúde, sendo convocada a revisar a sua prática e a elaborar novas estratégias para minimizar os impactos, como o estresse, gerados pela pandemia no atendimento aos pacientes com COVID-19. A exposição prolongada a fatores estressores, resulta no estresse ocupacional, que por sua vez contribui com o aumento da exaustão emocional e da despersonalização, assim como com a baixa realização profissional. Devido ao trabalho exaustivo e tenso, os profissionais de enfermagem estão mais propensos a desenvolver o estresse ocupacional (Barbosa et al., 2020; Marins et al., 2020).

Dada a crescente demanda relacionada à saúde mental nesse período, alguns países propuseram uma classificação da vulnerabilidade aos problemas psíquicos, elegendo como os mais susceptíveis aos problemas de saúde mental os profissionais da saúde, as pessoas infectadas, aquelas em isolamento devido à suspeita ou sintomas leves da doença e pessoas de contato próximo a estes. Os demais indivíduos do grupo de risco (idosos e pessoas com doenças crônicas) também devem receber apoio psicológico. Logo neste sentido, uma das estratégias que mais foram utilizadas por artistas e empresas nas mídias sociais tem sido a promoção das lives, que são transmissões online e ao vivo através de perfis públicos sobre diversos tipos de entretenimento (Moreira \& Lucca, 2020; Sousa Júnior et al., 2020).

\subsection{Produção Científica}

Com os avanços alarmantes dessa doença pelo mundo, cresce a necessidade de investimento em produções cientificas acerca da COVID-19. Atualmente vive-se em um século cuja informação é publicada e disseminada em grande escala e velocidade sem precedentes, tornando cada vez mais fácil o processo de incorporação por parte dos pesquisadores. A rapidez e 
a agilidade na produção de respostas não podem representar descumprimento das normas éticas, sanitárias e legais para pesquisas em seres humanos (Cheng et al., 2020; Costa et al., 2020; Silva et al., 2020).

As pesquisas cientificas representam a possiblidade de produzir conhecimentos que são de suma importância para promoção e proteção da saúde. Portanto, esta situação da pandemia é complexa, multifacetada e grave, impulsionando os profissionais de saúde a assumirem uma posição de ancoragem em evidências científicas e a buscar fontes confiáveis de difusão da informação. Diversas pesquisas têm sido desenvolvidas para a prevenção e para o controle da COVID-19, as quais têm se baseado nas lições aprendidas com outros surtos de doenças nas últimas décadas (Costa et al., 2020; da Silva et al., 2020).

Destaca-se que a maior produção científica advém do continente asiático, sobretudo da China, em que os profissionais de saúde de Wuhan contribuíram de forma significativa com o fornecimento de dados de pesquisa para a condução da assistência à saúde em todo o mundo. Tal fato ocorre em virtude de a referida cidade ter sido o primeiro epicentro da pandemia da Covid-19. A enfermagem aparece principalmente entre as categorias de profissionais no desenvolvimento dessas pesquisas com o compromisso de consolidar conhecimento no seu campo de atuação que possam contribuir simultaneamente para o crescimento e construção desse saber (Silva et al., 2020; Soares et al., 2020).

\subsection{Saúde das Gestantes, Recém-nascidos e Crianças}

Desde o início da pandemia da COVID-19, foi alertado sobre grupos populacionais considerados mais vulneráveis a infecção e com risco maior de evolução sendo eles, idosos, portadores de doenças crônicas, pacientes imunossuprimidos e imunodeprimidos, profissionais de saúde, gestantes e recém-nascidos. Embora existam evidências comprovando que em gestantes e crianças, exceto as oncológicas por se encontrarem em imunossupressão, o quadro grave da doença pode não desenvolver, os recém-nascidos permanecem nesse grupo devido seu sistema imunológico imaturo por estarem ainda em desenvolvimento (Pacheco et al., 2020; Rondelli et al., 2020).

Apesar de não terem incluso crianças no grupo vulnerável por não apresentarem quadros mais graves e muitas vezes adquirir a doença na forma assintomática, elas também precisam respeitar as ações adotadas para minimizar a transmissão do novo coronavírus, uma vez que elas se tornam veículo de transmissão para adultos, ocasionando assim um alto nível de estresse e ansiedade, se fazendo necessário que os pais observem tais mudanças de comportamento e busquem estratégias afim de diminuir os impactos nos hábitos sociais e de lazer causados pelo confinamento (Pacheco et al., 2020; Rondelli et al., 2020).

\subsection{Cloroquina e Hidroxicloroquina}

Nesse período de pandemia, abordagens não farmacológicas foram usadas como medidas de combate a disseminação do novo coronavírus, tal como a testagem dos casos assintomáticos e o isolamento de pessoas infectadas, porém mesmo assim observou-se o aumento da demanda e saturação nos serviços de saúde, em especial nos setores de cuidados críticos, uma vez que a infecção pelo Sars-Cov-2 pode resultar em um quadro grave, se fazendo necessário hospitalização ou internação em unidade de terapia intensiva podendo levar até a óbito. Diante disso, a busca por uma terapia antiviral segura e eficaz passou a ser uma emergência da pandemia, resultando na investigação de agentes antivirais por pesquisadores que começaram a explorar fármacos como a cloroquina, hidroxicloroquina e azitromicina, propostas como tratamento de surtos causados por coronavírus anteriores (Imoto et al., 2020; de Souza et al., 2020).

Em estudos in vitro foi possível comprovar atividade antiviral contra o Sars-Cov-2 dos fármacos hidroxicloroquina e cloroquina, tendo a hidroxicloroquina apresentado maior atividade antiviral (Imoto et al., 2020). Embora a literatura aponta que a cloroquina e hidroxicloroquina naturalmente estão associadas ao aumento de arritmias, caracterizando efeitos negativos, 
limitando assim a sua utilização para tratamento da COVID-19, fator esse que implica na necessidade de mais estudos (de Souza et al., 2020).

Já em estudos de fase pré-clínica foi apontado pouca ou nenhuma diferença no uso da hidroxicloroquina no tratamento e prevenção de pessoas expostas ou com risco de exposição ao vírus, bem como o uso da cloroquina que não expressou nenhuma diferença no tratamento ou recuperação clínica em pacientes acometidos pelo Sars-Cov-2, comprovando eficácia desconhecida contra o mesmo. Ainda vale salientar que um dos maiores questionamentos acerca da eficácia desses fármacos contra esse vírus diz respeito aos critérios e métodos dos estudos, principalmente em razão das amostras estudadas não representarem a condição real da pandemia (Singh et al., 2020).

\section{Conclusão}

A enfermagem é uma profissão que está ligada diretamente com o cuidado assistencial integral ao paciente, sendo uma das categorias de profissionais da saúde que mais se destacou dentro do cenário da pandemia, são os trabalhadores da saúde que mais estavam em contato com pacientes infectados pelo novo coronavírus, e diante desse cenário constitui-se o desafio da construção de conhecimento científico sobre essa doença.

A realização de pesquisas cientificas por profissionais ou acadêmicos de enfermagem se fez presente pouco tempo depois do início da pandemia, mesmo diante de tantos desafios que a COVID-19 trouxe para todos os profissionais da saúde, desafios maiores do que esses profissionais já estão acostumados a enfrentar no cotidiano, a categoria da enfermagem destinou tempo e dedicação para produzir conteúdo de caráter relevante para ciência diante das problemáticas enfrentadas na situação atual.

Se faz fundamental através de esforços conjuntos ressaltar que a enfermagem além do seu papel assistencial ainda desenvolve papel importante no meio cientifico que colabora para o avanço da ciência, os perfis das publicações dessa categoria apresentam uma preocupação não só com os impactos a saúde física para portadores da doença, mas também com os agravos que os resultados dessa pandemia trouxeram para a saúde mental, se preocupando assim de forma integral e holística com saúde de forma física, psíquica e social.

A fim que seja ressaltado a importância da cientificidade da profissão associando o pensar e o fazer, esse alicerce vem impulsionando novos patamares para a categoria que cada vez mais é reconhecida por sua ciência, abandonando aquela visão que a enfermagem limita-se a arte de cuidar. Sua contribuição através de pesquisas traz benefícios para a própria categoria e para a saúde em geral representando inovações e desenvolvimento.

Desta forma, se faz essencial que estudos futuros continuem sendo desenvolvidos nesta temática, pois é de importância para a saúde, impacta diretamente no bem-estar geral das pessoas, bem como para que cada vez mais a ciência e a literatura brasileira sejam incentivadas.

\section{Referências}

Barbosa, D. J., Gomes, M. P., \& Gomes, A. M. T. (2020). Fatores de estresse nos profissionais de enfermagem no combate à pandemia da COVID-19: síntese de evidências. Comun. ciênc. saúde. https://search.bvsalud.org/global-literature-on-novel-coronavirus-2019-ncov/resource/en/covidwho-525988

Botelho, L. L. R., de Almeida Cunha, C. C., \& Macedo, M. (2011). O método da revisão integrativa nos estudos organizacionais. Gestão e sociedade, 5(11), $121-136$.

Cheng, C. et al. (2020). Comportamento da produção científica sobre síndrome respiratória aguda grave. Revista Ciências da Saúde. https://preprints.scielo.org/index.php/scielo/preprint/view/288

Costa, I. C. P. et al. (2020). Produção científica em periódicos Online sobre o novo coronavírus (COVID-19): pesquisa bibliométrica. Texto \& ContextoEnfermagem, 29.

Dantas, T. P., de Aguiar, C. A. D. S., Rodrigues, V. R. T., da Silva, R. R. G., da Silva, M. I. C., Sampaio, L. R. L., \& Pinheiro, W. R. (2020). Diagnósticos de enfermagem para pacientes com COVID-19. Journal Health NPEPS, 5(1), 396-416. 
da Silva, V. R. F. et al. (2020). Análise bibliométrica da produção científica sobre Coronavírus e Covid-19. Saúde Coletiva (Barueri), 10(53), 2356-2369.

de Lima, T. J. A., de Lima, M. V. C., de Oliveira, K. K. D., \& Ferreira, V. O. (2020). A Imagem do Enfermeiro no Instagram no Contexto da Pandemia da Covid-19. Enfermagem em Foco, 11(1. ESP).

de Souza, J. B., Cavalcanti, I. D. L., de Lacerda, D. C., Soares, J. C. S., \& de Oliveira, F. H. P. C. (2020). Hidroxicloroquina como alternativa para o tratamento da infecção causada pelo SARS-CoV-2: O que se sabe até agora? Brazilian Journal of Health Review, 3(3), 4255-4273.

de Sousa Júnior, B. S. et al. (2020). Pandemia do coronavírus: estratégias amenizadoras do estresse ocupacional em trabalhadores da saúde. Enfermagem em Foco, 11(1. ESP).

de Sousa Neto, A. R., \& de Freitas, D. R. J. (2020). Utilização de máscaras: indicações de uso e manejo durante a pandemia da covid-19. Cogitare Enfermagem, 2.

do Nascimento, V. F., Espinosa, M. M., da Silva, M. C. N., Freire, N. P., \& Terças-Trettel, A. C. P. (2020). Impacto da COVID-19 sob o trabalho da enfermagem brasileira: aspectos epidemiológicos. Enfermagem em Foco, 11(1. ESP).

Forte, E. C. N., \& Pires, D. E. P. D. (2020). Os apelos da enfermagem nos meios de comunicação em tempos de coronavirus. Revista Brasileira de Enfermagem, 73 .

Gandra, E. C., da Silva, M. F., da Silva, E. S. T., Regly, I. C. V., \& Silva, C. M. R. (2020). Fatores de riscos assistenciais relacionados a contaminação de profissionais de enfermagem por Covid-19: Uma revisão da literatura. Brazilian Journal of Development, 6(7), 53348-53360.

Geremia, D. S., Vendruscolo, C., Celuppi, I. C., de Souza, J. B., Schopf, K., \& Maestri, E. (2020). Pandemia COVID-2019: formação e atuação da enfermagem para o Sistema Único de Saúde. Enfermagem em foco, 11(1. ESP).

Imoto, A. M. et al. (2020). Cloroquina e Hidroxicloroquina no tratamento da COVID-19: Sumário de Evidências. Comunicação em Ciências da Saúde.

Machado, D. M. et al. (2020). Parada cardiorrespiratória na pandemia por coronavírus: revisão compreensiva da literatura. Revista Enfermagem UERJ, 28, 50721 .

Marins, T. V. O. et al. (2020). Enfermeiro na linha de frente ao COVID-19: A experiência da realidade vivenciada. Research, Society and Development, 9(8), e710986471.

Moreira, A. S., \& de Lucca, S. R. (2020). Apoio psicossocial e saúde mental dos profissionais de enfermagem no combate ao COVID-19. Enfermagem Em Foco, 11(1. ESP).

Pacheco, S. T. A., Nunes, M. D. R., Victória, J. Z., da Silva Xavier, W., da Silva, J. A., \& Costa, C. I. A. (2020). Recomendações para o cuidado à criança frente ao novo coronavírus. Cogitare Enfermagem, 25.

Pedrosa, N. L., \& Albuquerque, N. L. S. D. (2020). Análise Espacial dos Casos de COVID-19 e leitos de terapia intensiva no estado do Ceará, Brasil. Ciência \& Saúde Coletiva, 25, 2461-2468.

Rondelli, G. et al. (2020). Assistência às gestantes e recém-nascidos no contexto da infecção COVID-19: uma revisão sistemática. DESAFIOS-Revista Interdisciplinar da Universidade Federal do Tocantins, 7(Especial-3), 48-74.

Schneider, G., Bim, F. L., Sousa, Á. F. L. D., Watanabe, E., Andrade, D. D., \& Fronteira, I. (2021). Utilización de textiles impregnados con antimicrobianos en los servicios sanitarios: revisión integradora. Revista Latino-Americana de Enfermagem, 29.

Singh, B., Ryan, H., Kredo, T., Chaplin, M., \& Fletcher, T. (2020). Chloroquine or hydroxychloroquine for prevention and treatment of COVID-19. The Cochrane database of systematic reviews, 2020(4).

Silva, D. P., dos Santos, I. M. R., \& Melo, V. S. (2020). Aspectos da infecção ocasionada pelo Coronavírus da Síndrome Respiratória Aguda Grave 2 (SARSCoV-2). Brazilian Journal of Health Review, 3(2), 3763-3779.

Silva, R. E. D., Sallas, J., Novaes, L. C. G., \& Guilhem, D. B. (2020). Desafios na condução de pesquisas sobre a COVID-19 envolvendo seres humanos no Brasil. Comun. ciênc. Saúde, 48-60.

Soares, S. S. S. et al. (2020). Pandemia de Covid-19: Pesquisa documental a partir de publicações do Conselho Federal de Enfermagem. Enfermagem em Foco, 11(1. ESP).

Sousa Júnior, J. H., de Sousa, L. V. H. A., Santos, W. S., Soares, J. C., \& Raasch, M. (2020). "\# Fiqueemcasa e cante comigo": estratégia de entretenimento musical durante a pandemia de covid-19 no brasil. Boletim de Conjuntura (BOCA), 2(4), 72-85.

Souza, M. T. D., Silva, M. D. D., \& Carvalho, R. D. (2010). Revisão integrativa: o que é e como fazer. Einstein. 8(1), $102-106$. 